\title{
78 \\ Tree Structure Operation for Video Editing Utilizing Image Recognition Technology
}

\author{
Hirotada Ueda \\ Research \& Development laboratory, \\ Hitachi Denshi, Ltd. \\ 32 Miyuki-cho, Kodaira, \\ Tokyo 187, Japan \\ hiro-u@po.iijnet.or.jp
}

\author{
Takafumi Miyatake \\ Central Research Laboratory, \\ Hitachi, Ltd. \\ 1-280 Higashi-koigakubo, Kokubunji, \\ Tokyo 185, Japan \\ miyatake@crl.hitachi.co.jp
}

\begin{abstract}
This paper proposes an approach to integrate image recognition technology into nonlinear video editing system and discusses the results of a prototype development. The prototype consists of five support functions for video editing; automatic scene separation during disk recording, tree structure story editing, quick review with micon, film metaphor interface and integrated information management. The automatic scene separation is the exact solution for the complaint of the current system users. The tree structure story editing and the integrated information management provide methods to author video structure in several different detail levels and relieves the constrain of limited size of the window. The quick review with micon is a convenient shot comparison method. The film metaphor interface offers a intuitive and speedy operation for video strings.
\end{abstract}

Keywords video editing, video structure, film metaphor, scene separation, image recognition

\section{INTRODUCTION}

Multimedia techriology is a rapidly growing one. One of the typical applications is a nonlinear video system. In the nonlinear video system hard disk recording technology offers a quick and random access capability to handle video contents. This fact removes loss time of tape winding a lot. Computer technology also offers good GUIs (Graphical User Interface).

Another feature of a nonlinear video system is the capability of combine different type of information in a integrated manner [1]. Keywords and annotations and so on are easily attached and managed in a efficient way. These are grate advantage when comparing with previous tape oriented editing systems. However, in current nonlin- ear video systems, computers seem to be used only as controllers for the hard disk devices or as generators for GUIs. We do not yet use full computing power of data processing effectively in this field. Consequently video handling is still time consuming work and needs trained operators.

Recently an approach combining image recognition technology with multimedia technology is getting popular [2]. There are two application types in this approach; editing [3] [4] and archives [5] [6] [7] [8]. In these applications temporal-spacial video contents structure is automatically analyzed and the results are used to help users handle video contents. Functions utilized image recognition technology offer descriptions of scene separations, motion of the camera and filmed objects, tracks and

Human-Computer Interaction: INTERACT'97 S. Howard, J. Hammond \& G. Lindgaard (editors) Published by Chapman \& Hall OIFIP 1997 
contour lines of objects, existence of objects, periods of existence and automatic linking of identical objects.

There are many good research results. However there are few real products. Are those not so attractive nor usable for users? One of the reason is the fact that integration of those functions has not been sufficient. In this paper we propose new design concepts to integrate those and utilize full computing power for the nonlinear video editing. We also show the results of our prototype system.

\section{DESIGN CONCEPTS}

Through the discussion with the users of current nonlinear editing system, we found that they have a common complaint. That is a manual scene cutting process as a preparation for the nonlinear icon based editing. Even after the long waiting time for digital copy recording, they cannot start editing yet. They first have to carefully watch the contents and choose the point to cut scenes.

Another users request for more effective video editing is a method that can show the total video structure in several levels of detail seamlessly.

Because of the similarity with film based editing, where they can really directly move a shot very quickly, users who are familiar to the computer like the icon based editing very much. However motion picture oriented users complain that such a operation is a directly imported one from the computer world.

Users also require a good method to manage informa- tions that are generated in the video editing process from shooting, editing to the end of archiving stage. Huge amount of informations are generated. Typical examples are dates, still pictures, written/voice memos, contract conditions, copy rights information and so on. They are valuable but cannot be stored with the video in current systems.

To relief these users inconveniences, we developed following five new functions:

- Automatic scene separation during disk recording

- Tree structure story editing

- Quick simultaneous shots review

- Film metaphor interface

- Integrated multimedia information management

We had developed the automatic scene separation technology in 1990[2]. At that moment the power of workstation is not sufficient and we could just do non-real-time scene separation and hard disk recording separately. However now we have more CPU power even with a smallest (cheapest) workstation. We also found that some workstation has multiple video data paths that can be used to achieve real-time scene separation during compression recording. This is a main issue to answer the above users complaint. This feature removes time consuming manual separation process and user can immediately start editing after the hard disk recording.

Our solution for a effective video editing is the tree structure story editing. We employ the combination of the tree structure and links between objects. Figure 1 shows this structure. Here the scenario is an entire story composed of a number of scenes. Each scene consists of one

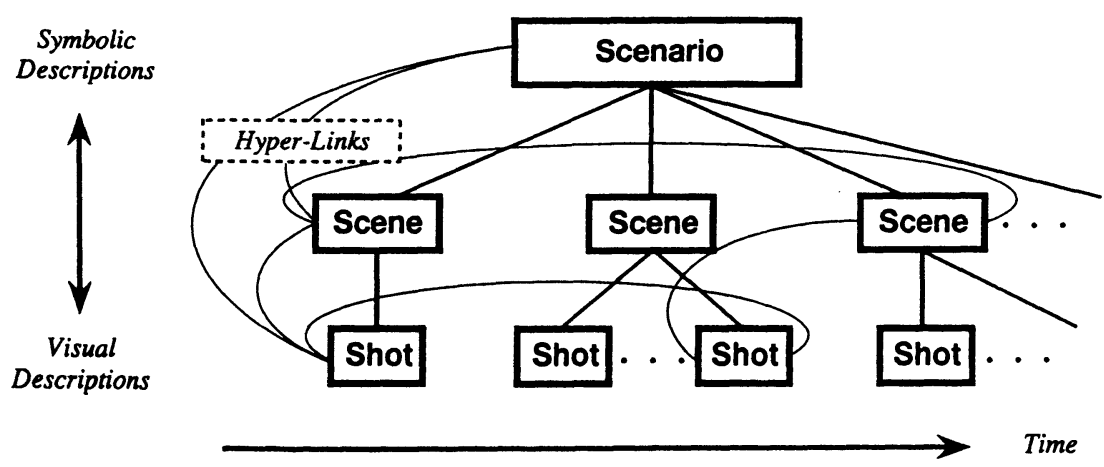

Figure 1. Tree Structure of Video 
or more shots. Scenes may have sub-scenes. In this tree structure, the upper parts have symbolic descriptions and the lower parts have more visual descriptions. The lowest parts (i.e., shots) of this structure contain raw video data. According to this scheme we designed a new GUI that enables the user to easily understand the video structure and to edit story through this visual structure. Using this tree structure the user can handle video contents in any size of blocks like chapters, sections, paragraphs and words in a book.

One important idea of this design is a capability to partly hide this structure. For instance, when some scene is already edited enough, the user hides the lower part of this scene and can concentrate upon the rest of the story. This technique offers a efficient use of the limited computer screen size.

We also add a convenient method to check the contents of each shot. We borrow the idea of micon (moving icon) from Davenport group [9]. Using this technique the user can view the miniature size play back of multiple shots simultaneously. This method gives the user, for example, a capability of quick comparison of shots to select the best one.

We introduced the film metaphor interface in the special effect operation. Most TV station people said, this operation, utilizing simple thumbnail and time line editing window, was one of the inconvenient part of current systems.

To achieve the integrated multimedia information management we found that the tree structure for editing story is good to store these informations on it. Any information could be attached to the objects on the arbitrary level of the tree depending on its nature.

\section{PROTOTYPE}

\subsection{Hardware}

Hardware specification of our prototype system is the followings:

- Low cost off the shelf workstation

(SGI INDY with Indy Video)

- Motion JPEG compression board

(SGI Cosmo Compress).

To achieve the simultaneous operation of automatic scene separation and compression recording, we assign two video capturing paths; VINO (originaly equipped) and Indy Video (optional one). Using the VINO video path we get a miniaturized image of each frame and compare a pair of these images frame by frame to detect scene change points. When a scene change point is detected, the system records the time code of this frame. At the same time all miniaturized images are stored into the hard disk. These images are used for the micon play back. The video data given through the Indy Video path is feed into the Cosmo Compress and compressed video is recorded into the hard disk.

\subsection{Software}

The size of the software of our prototype system is about $90 \mathrm{~K}$ steps. The language is C. Operating system IRIX and control software for SGI hardware are the normal one came with the hardware. Therefore this software is easily transported to the SGI family systems.

\subsection{Functions}

Our prototype application program is a nonlinear video editing system that has five new functions. Figure 2 shows a typical screen image of the system.

\section{Automatic scene separation}

There is no special operation for the Automatic scene separation. When the user set up the video source and start the hard disk recording in the same manner as current systems, our system automatically start the Automatic scene separation. This process runs simultaneously with the digital compression and hard disk recording process. During the recording a monitor window opens in the center of the screen.

\section{Tree structure story editing}

The lower window in Figure 2 is a pool of shots. After the digital recording process, automatically separated shots are visually listed in this window. Each micon is displayed as a box with a depth which indicates the time length of the shot as shown in Figure 2. This representation compactly visualizes the temporal structure of the video. Then the user chooses appropriate shots and drugs them into upper window.

The upper window is an editing area. Here we introduced the tree structure story editing. The user connects several shots into a block as shown in Figure 2. This operation makes a pert of the tree. When shots are 


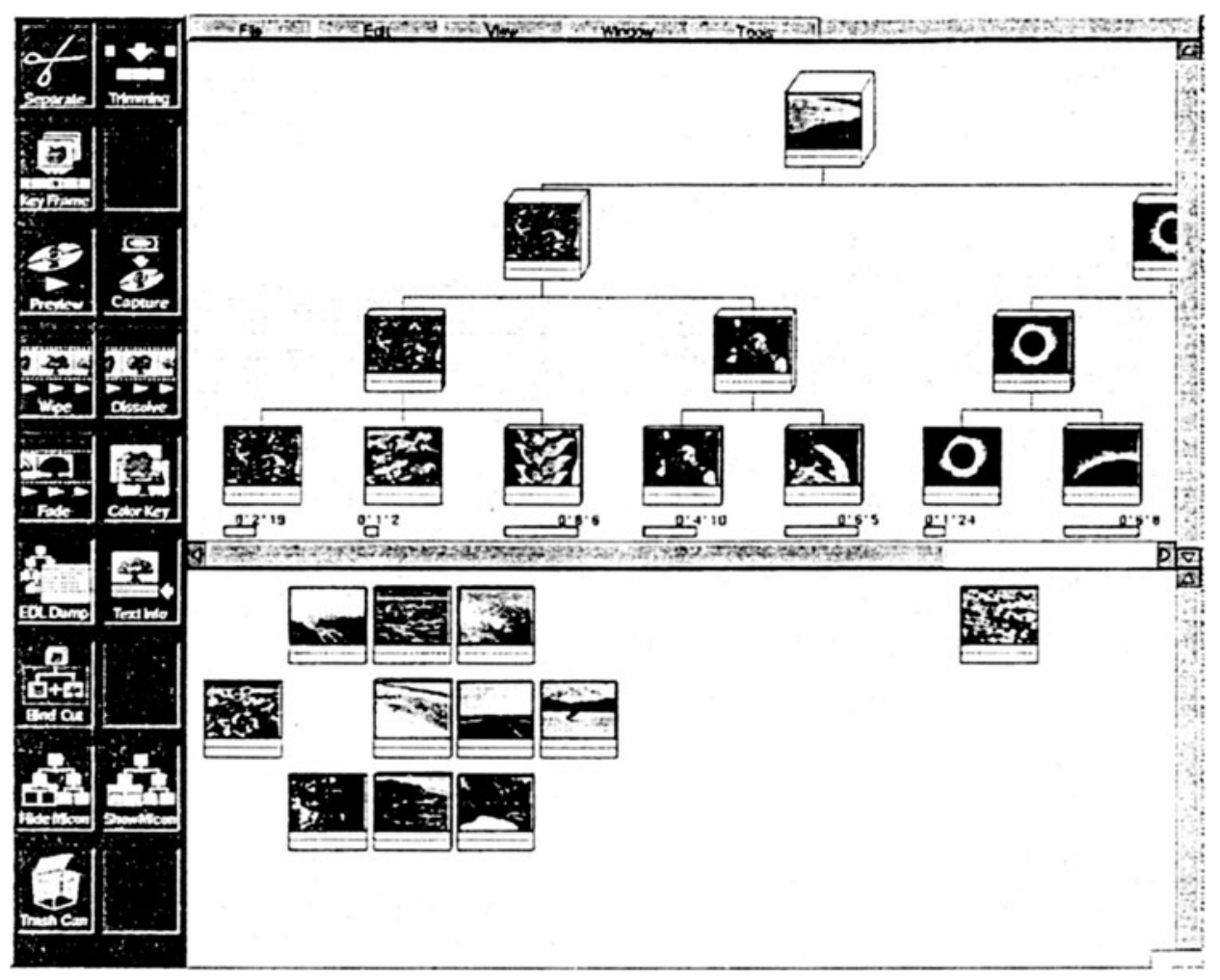

Figure 2. A screen of the prototype system

connected a new higher layer micon is generated. The user repeat such steps and create whole tree structure. This structure corresponds to the one shown in Figure 1. Time axis is left to right and logical abstraction level is bottom to top.

Raw video data (shot) is located at the lowest level of the tree. The user can attach annotations to any level of icons using the dialogue windows. The abstraction level of these annotation might differ according to the height of the tree structure. All micons has a thumbnail image of the first frame of the shot or the group of the shots by default setting. The user can change this with any frames within the duration of the shot or the group of the shots.

While editing, the user moves shots one by one along the time axis as a usual editing system. The user also can move a block of shots with a single action by picking the upper level icon of the tree. Using this function the user, for

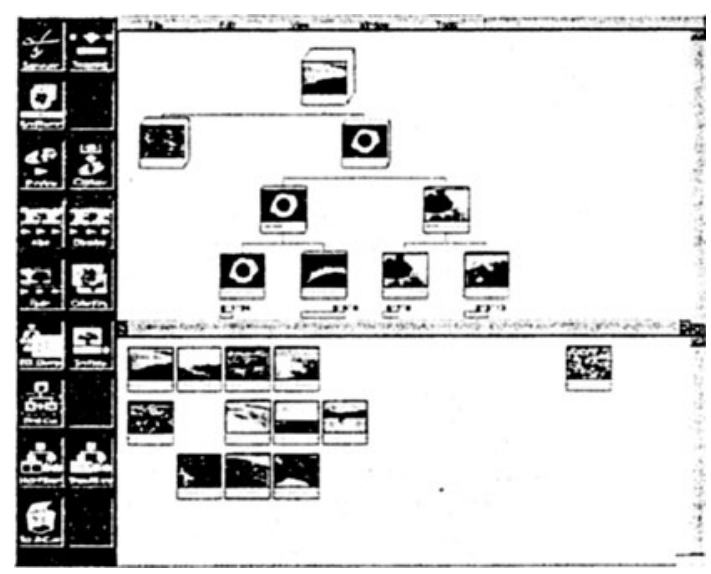

Figure 3. A screen of the hidden tree 


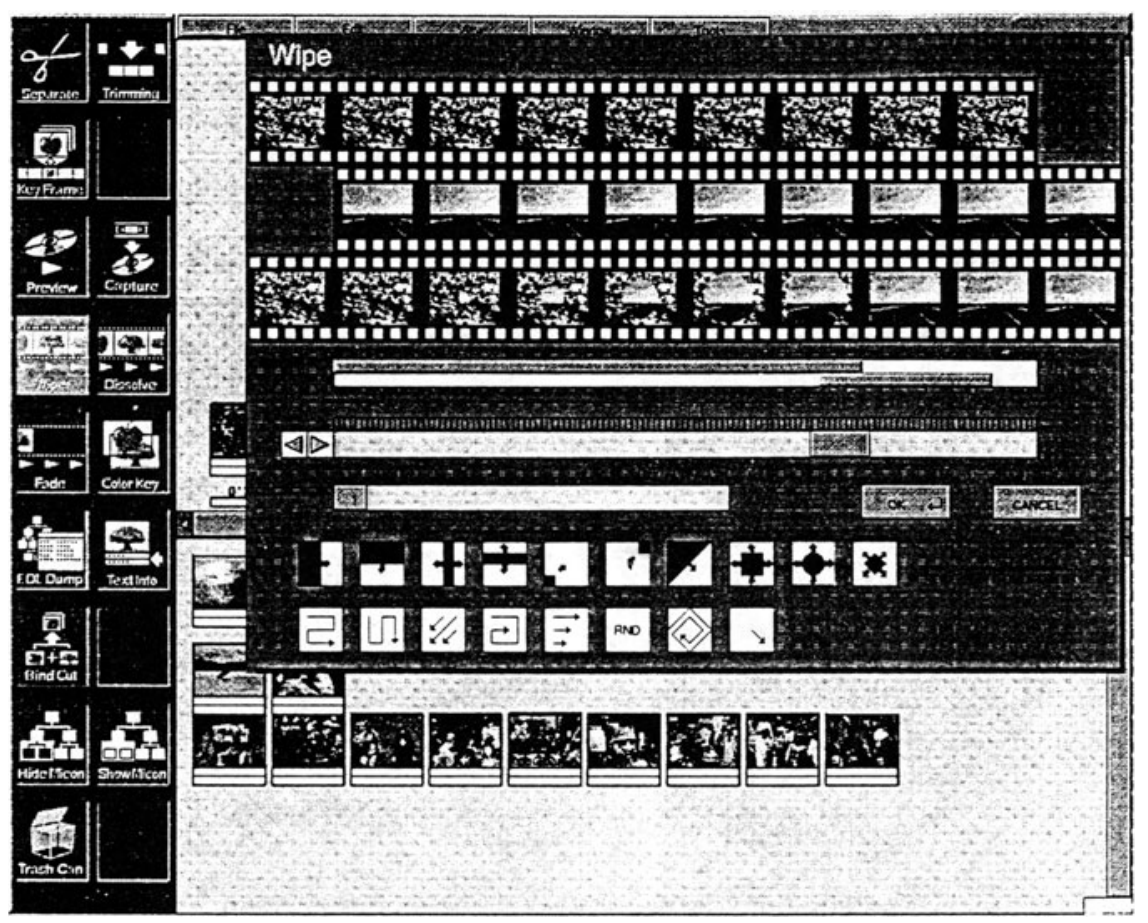

Figure 4. A screen of the special effect operation

instance, can easily change the order a scene consists of flower shots and a scene of mountain shots. In both cases, when a icon moved to any place at the same level of the tree the order of the block is changed.

When the user think that some block is well constructed and want to concentrate in the rest of the story, the user select the top icon of that block and hide the lower part of the block. This situation is shown in Figure 3. The block at the left side in Figure 2 is hidden and a right side block (those are out of the window and not visible in Figure 2) comes into the window in Figure 3. Thus the user overcomes the problem of the limited area of the window.

\section{Quick simultaneous shots review}

Shots are presented as micons so that the user can play back arbitrary numbers of them simultaneously in any time without the operation to open monitor windows. Therefore the user can check the content within a structure at a glance.
The size of a miniaturized video frame is only $80 \times 60$ pixels ( $15 \mathrm{~K}$ byte) instead of $640 \times 480$ of a full frame. This size is fairly less than a compressed frame (around $100 \mathrm{~K}$ byte). Father more miniaturized video frames does not require decompression that cause some overhead to fill the buffer memory etc. In fact the play back of micon is immediate but play back of compressed video has 0.5 to 1 seconds idle time after the users button operation in our prototype. Micon play back is very light process for the computer and we can play even ten or more micons simultaneously. This is a big benefit for the user when the user compare multiple similar shots.

\section{Film metaphor interface}

The miniaturized video frames also offer a good function for the user interface of special effect operations. Figure 4 shows an example. This is a situation when the user does a wipe effect. A wipe operation window is opened in the center of the screen. Here the upper film metaphor shows 


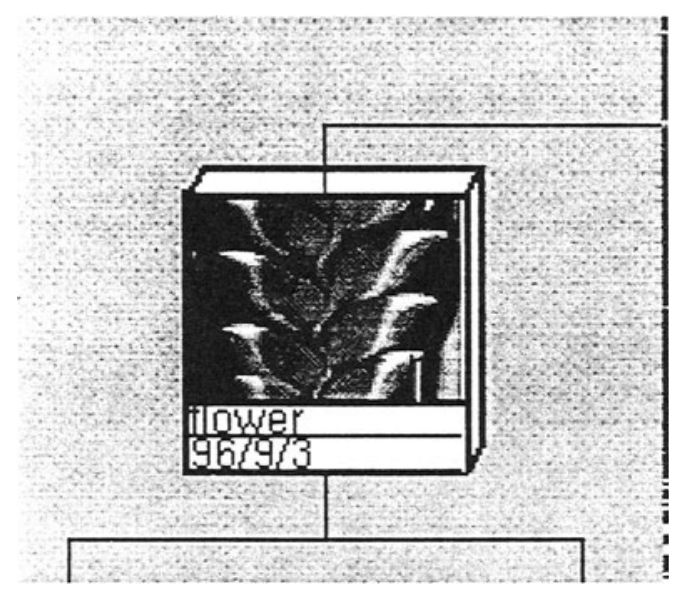

Figure 5. A close up of the micon with annotation

an A-roll video shot, the middle shows a B-roll shot and the lower film metaphor shows a result of a wipe effect. The set of scroll bars under the film metaphors controls the overlap amount of A and B-roll. The simple scroll bar below them controls the viewing position. Using the lowest slider the time resolution can be changed by the correspondence between one film flame and the number of the video flames.

As the size of miniaturized frames is fairly small, regeneration of result film metaphor according the change of the effect pattern is immediate. When we generate it using compressed video it requires about three times of a real time before showing the special effect result. The operation of the overlap amount is also as smooth and intuitive as real film handling.

\section{Integrated multimedia information management}

We provide a dialog box for each nodes of the tree structure. Thorough the dialog box the user can attach any information to the appropriate nodes. Any kind of multimedia information can be linked to any nodes using this dialog box. Therefore the user can manage all information related to the video in a layered structure very easily. Some portion of the information selected by the user (e.g. date, the title of the section of the story etc.) can be shown in the lower part of the micon as shown in Figure 5.

\section{DISCUSSION}

Through the evaluation of earlier version of our prototype that has not the simultaneous scene separation nor the tree structure story editing [3], we found that visual listing of video structure is efficient support for video editors. Conventional video editing system requires numerous repetitions of fast-forward and rewind operations until an overview of the structure, as shown in the components window of Figure 3, is firmly fixed in the user's mind; this is very time consuming. Image recognition technology removes this time-consuming step in video editing.

To answer the further users request we realized a simultaneous scene separation during the hard disk recording. Professional users are very much sensitive to the accuracy of separation point (a frame rate accuracy is required). If processing time is not quick enough, the system cannot catch the accurate timing of the scene change. So we have to perform frame by frame image comparison in every 33 msec. We had to try many fine tuning of our program to achieve this functionality. Finally we found that this program uses almost full computing power and data transfer capability of the newest workstation (that is the lowest cost one though). The recognition accuracy is also important issue. The total error rate of our scene separation is less than $1 \%$.

Miniaturized video frames are valuable to improve the system performance. Their data size are fairly small and do not use complicated compression. Consequently they do not require heavy process. However they efficiently offer intuitive visual interface such as the special effect operation interface and the film metaphor interface.

We have just finished our prototype and we did not have field evaluations yet. However when we show the demo, almost all users of current nonlinear editing system say that the simultaneous scene separation is really valuable. They also say the tree structure story editing with the hidden lower structure and the integrated multimedia information management attached to this tree structure may fit in drama and documentary video editing well.

As described earlier we developed our prototype on the standard hardware and system software. So it is easily transported to the any SGI family systems. One exception is the simultaneous automatic scene separation. If the system does not have dual video paths, this is not possible. Further if the control software for the video paths given by the hardware vender is not API compatible, some part of 
our program needs modification. In fact we now has two versions, one for INDY and the other for Indogo2. But the Indigo 2 version does not have the simultaneous automatic scene separation capability because we could not find the way to open two video paths in parallel.

Through the experience of this development we strongly request more powerful standard multimedia data paths in the workstation (also PC) as well as the power of the CPU. When we get more powerful data paths and CPU we can produce more intelligent functions that work simultaneously to analyze video contents in various point of view. Then we can create more usable nonlinear video systems.

\section{CONCLUSION}

We proposed a new approach to design a nonlinear video editing system. It consists of five funtions; Automatic scene separation during disk recording, Tree structure story editing, Quick simultaneous shots review, Film metaphor interface and Integrated multimedia information management. According these concepts we have developed a prototype. The automatic scene separation is the exact solution for the complaint of the current system users. This was the most difficult one and we use almost full computing power and data transfer capability of the newest workstation. The tree structure story editing provides a convenient method to edit video structure in several different detail levels. It also offers a way to change the order of a group of shots quickly. The hidden lower structure technique also relieves the constrain of limited size of the window. The quick review with micon gives the user a convenient shot comparison method. Miniaturized video frames also achieves a speedy and intuitive interface for the special effect operation. The integrated multimedia information management method utilizing the tree structure is powerful tool to maintain informations generated through out the video creating procces.

\section{REFERENCES}

[1] Davenport, G., Aguierre-Smith, T. and Pincever, N. "Cinematic primitives for multimedia," IEEE Computer Graphics and Applications, 11(4), pp. 67-75, (1991).

[2] Ueda, H., Miyatake, T. and Yoshizawa, S. "IMPACT: An Interactive Natural-Motion-Picture Dedicated Multimedia Authoring System," ACM CHI'91; pp. 343-350 (1991).

[3] Ueda, H., Miyatake, T., Sumino. S. and Nagasaka, A. "Automatic Structure Visualization for Video Editing," ACM INTERCHI'93, pp. 137-141 (1993).

[4] Mackay, E. W. and Davenport, G. "Virtual Video Editing in Interactive Multimedia Applications," Communications of the ACM, 32(7), pp. 802-810 (July 1989).

[5] Nagasaka, A., and Tanaka, Y. "Automatic Video Indexing and Full-Video Search for Object Appearances," IFIP WG 2.6 2nd Working Conference on Visual Database Systems, pp. 119-133 (1991).

[6] Zhang, H. J., Low, C. Y., Smoliar, S. W. and Wu, J. H. "Video Parsing, Retrieval and Browsing: An Integrated and Content-Based Solution," ACM Multimedia'95, pp. 15-24 (1995).

[7] Taniguchi, Y., Akutsu, A., Tonomura, Y., and Hamada, H. "An Intuitive and Effisient Access Interface to Real-Time Incoming Video Based on Automatic Indexing," ACM Multimedia'95, pp. 2533 (1995).

[8] Smith, M. A., Christel, M. G. "Automating the Creation of a Digital Video Library," ACM Multimedia'95, pp. 357-358 (1995).

[9] Brendmo, H. P. and Davenport, G. "Creating and viewing the elastic Chrals - a hypermedia jarnal," Hypertext. State of the art. McAlesse, R. and Greene, C. (eds.), Intellect Ltd. (1990). 